\title{
Interictal autonomic dysfunction in patients with epilepsy
}

\author{
Kanar K. Shaker ${ }^{1}$, Akram M. Al Mahdawi² and Farqad B. Hamdan ${ }^{3^{*}}$ (D)
}

\begin{abstract}
Background: Autonomic nervous system (ANS) symptoms are frequently present in people with epilepsy (PwE). They are generally more prominent when they originate from the temporal lobe. We aim to investigate the alterations of autonomic functions during the interictal period in patient with temporal lobe epilepsy (TLE) and idiopathic generalized epilepsy (IGE) using heart-based tests, blood pressure (BP)-based tests and sympathetic skin response (SSR). Forty-eight PwE with disease duration ranging from 2 to 15 years and 51 healthy individuals were studied. Long-term electroencephalography (EEG) monitoring, the heart rate variability (HRV) during normal breathing, deep breathing, Valsalva maneuver and standing, BP responses during standing, to isometric hand grip and to mental arithmetic, and the SSR was recorded for all participants.

Results: 31 patients with TLE and 17 with IGE showed lower RR-IV values during deep breathing, Valsalva maneuver and standing, but not during rest, impaired BP responses during standing, isometric hand grip, and mental arithmetic. Also, prolonged SSR latencies. Within PwE group, no difference was noticed between males and females, nor between the left and right temporal lobes.

Conclusion: Abnormal autonomic (sympathetic and parasympathetic) regulatory functions suggest that epilepsy may alter the autonomic function and this is not only in TLE but rather in IGE too. These autonomic changes are irrespective of the localization of epilepsy between the two hemispheres. The ANS changes in epileptic patients, particularly those with autonomic symptoms, confirm that electrophysiologic measures of autonomic function may be of value in preventing sudden unexpected death in epilepsy.
\end{abstract}

Keywords: Epilepsy, Autonomic function tests, Sympathetic skin response

\section{Background}

Epileptic seizures are frequently accompanied by autonomic symptoms either as complement to other seizure symptoms or as the principal seizure manifestation. They are mediated by an activation of the central autonomic network (CAN) [1]. The CANs implicated in the pathophysiology of autonomic epilepsies are primarily represented in the non-dominant hemisphere, thus autonomic

\footnotetext{
*Correspondence: Farqadbhamdan@colmed-alnahrain.edu.iq; farqadbhamdan@yahoo.com

${ }^{3}$ Department of Physiology, College of Medicine, Al-Nahrain University, P.O. Box 70044, Baghdad, Iraq

Full list of author information is available at the end of the article
}

findings are common in temporal lobe epilepsies (TLE) and insular lobe epilepsies [2].

Autonomic symptoms can be seen before, during or after seizure including cardiovascular changes, respiratory manifestations, gastrointestinal symptoms, cutaneous manifestations, pupillary symptoms, genital and sexual manifestations as well as urinary symptoms $[3,4]$.

They may be caused by overactivity of the sympathetic NS, even though there is some contribution of the parasympathetic NS, especially symptoms of cardiovascular autonomic dysfunction [5].

Certain autonomic symptoms may provide lateralizing and sometimes localizing information on the seizure onset zone, although some of these signs may appear as a result of discharge spreading [6]. 
Autonomic signs and symptoms can signify the main symptom at the start of a focal seizure, then categorized as a focal onset autonomic seizure. Conversely, clinically pertinent autonomic symptoms and signs accompany seizures of focal, generalized, and/or unknown onset, the seizure is categorized according to other, more related features [1].

Autonomic symptoms range from slight seizure manifestations to severe life-threatening events such as postictal generalized electroencephalographic (EEG) suppression and transient episodes of apnea and bradycardia terminating in a fatal asystole [6]. PwE, particularly those with long-standing uncontrolled seizures, have measurable alterations of resting autonomic function. These alterations may be relevant to the increased risk of premature mortality in epilepsy, including sudden unexpected death in epilepsy [7].

Epilepsy may also cause functional ANS changes, including a decrease in heart rate variability (HRV) [4]. Previous studies based on short or long-term ( $24 \mathrm{~h}$ ) electrocardiographic recordings revealed decreased HRV in patients with chronic epilepsies, mainly in peri-ictal period [8-10].

We aimed to investigate cardiovascular autonomic and sudomotor function in TLE and idiopathic generalized epilepsy (IGE) in comparison to control subjects, whether there is any different behavior in autonomic function tests of PwE based on their gender, and to look for any association between the autonomic changes (if any) and the lateralization of epileptic seizures.

\section{Methods}

A case-control study conducted at the neurophysiology Unit, Baghdad Teaching Hospital and Al-Imammain AlKadhimiyain Medical City for the periods from May 2019 to November 2020. Ethical approval was obtained from the Iraqi Council of Medical Specialization (Decision No. 931: date: 1/3/2020). Written informed consent was also granted by all participants.

A total of $57 \mathrm{PwE}$ diagnosed based on clinical neurological examination, EEG findings, and 3-Tesla brain magnetic resonance imaging (MRI) were studied. The duration of their illness ranged from 2 to 15 years. They were classified according to the ILAE 2007 to have either TLE or IGE. Five patients with brain structural abnormality on MRI, one pregnant female, one patient with status epilepticus of $<15 \mathrm{~min}$, and two patients with recent convulsions within $48 \mathrm{~h}$ before the study were excluded.

The remaining $48 \mathrm{PwE}$ were with uncontrolled seizure frequency ranged from 1 attack/month to 1-3 attacks/ week and the EEG records show interictal temporal spikes/sharp, spike/sharp waves discharges, and generalized spike wave or poly spike wave with the normal background. They were on two or more appropriately chosen and used antiepileptic drugs (AEDs) including carbamazepine, sodium valproate, levetiracetam, lamotrigine, and ethosuximide. None of them was refractory to treatment. All were free from any disease that may affect the ANS and cerebral blood flow, including polyneuropathies, multiple sclerosis, diabetes mellitus, Parkinson's disease, heart disease, hypertension, and central NS infection or head trauma). Another 51 age- and sexmatched subjects serve as controls were studied.

Study participants were told to abstain from food, alcohol, coffee, cola, energy drinks, tea, and tobacco, and ANS-altering drugs at least for $4 \mathrm{~h}$ on the scheduled test day [11] and to avoid activities that would affect BP (like running and jumping) for $2 \mathrm{~h}$ before the tests [12]. The patients are advised to wear comfortable clothes and to shower the night before testing without using anybody lotions, powders, or creams below the neck, also to drink water and stay hydrated.

Before the autonomic function tests were conducted, each subject's medical history, demographic data, and physical examination findings were recorded.

To evaluate ANS functions, SSR, HRV (R-R interval variation), and BP changes were measured using Micromed (Italy) and Medtronic Keypoint (Denmark) 4-channel electromyography equipment. The tests were conducted in a quiet room at room temperature of $22 \pm 2{ }^{\circ} \mathrm{C}$. A surface recording disk electrode fixed to the left anterior chest area at the fourth and fifth intercostal space and a reference electrode is fixed at the left anterior axillary line over the fifth or sixth rib. The ground electrode was placed on the midline of the sternum. The sensitivity and sweep speed are adjusted to display the QRS complexes on the screen. HRV analyses are based on the measurement of the time intervals between successive QRS complexes, which reflect the regulation of the HR by the ANS via its sympathetic and parasympathetic control mechanisms [13]. This means that HRV analyses can be used to provide indirect clues about NS activity.

The test battery included recording of HR responses at rest (normal breathing), deep breathing, Valsalva maneuver, and standing as well as measurements of BP changes during isometric handgrip, after 3-min standing, and mental stress test.

The HR response to deep breathing was expressed as deep breathing difference, which was the difference between the maximum HR (shortest RR interval during inspiration) and the minimum HR (longest RR interval during expiration), in a patient breathing at six cycles per minute. The exhalation:inspiration (E:I) ratio was obtained using the following formula: the RR-IV\% $=$ (the longest $R R$ - the shortest $R R) \times 100 /$ mean of $R R$ values [14]. 
The HR response to Valsalva maneuver expressed as Valsalva ratio was tested while the patient in supine position and the head slightly elevated to about $30^{\circ}$. The patient was asked to strain against $40 \mathrm{mmHg}$ for $15 \mathrm{~s}$ by blowing into a mouthpiece attached to a sphygmomanometer. The ratio of the longest RR interval 30-45 s following the release of strain to the shortest $R R$ interval during strain was calculated. The minimal HR occurs at 15-20 s after releasing the strain [15].

$\mathrm{HR}$ response to standing was obtained after the patient has been resting at least for $20 \mathrm{~min}$. It is expressed as the $30: 15$, which is the ratio of the longest RR interval (slowest HR) at $30 \mathrm{~s}$ to the shortest RR interval (fastest HR) at $15 \mathrm{~s}$, following an abrupt change in position from supine for $3 \mathrm{~min}$ to standing. The 30/15 ratio should be at least 1.04 [16].

Changes in systolic BP following change of position from supine to standing after 3 min were also calculated. The postural decrease in BP after 3 min was taken as the difference between BP (systolic and diastolic BP) lying and the BP (systolic and diastolic BP) standing. A decline in systolic BP by more than $20 \mathrm{mmHg}$ and by more than $10 \mathrm{mmHg}$ for diastolic BP is considered abnormal [15].

The isometric handgrip test involved periodic BP recordings (1-min interval), whereas subjects contracted a hand dynamometer at $30 \%$ of maximum voluntary contraction for $4 \mathrm{~min}$. The difference between the highest diastolic BP just before the contraction was released, and the handgrip started was taken as a measure of the response. It should normally be more than $15 \mathrm{mmHg}$ [15].

The systolic BP response to mental stress test was calculated when the patient performs mental arithmetic calculations by removing the number 7 successively from 100. The increase in systolic BP should exceed $10 \mathrm{mmHg}$ in normal individuals [15].

When measuring SSR, an active $\mathrm{Ag} / \mathrm{AgCl}$ disk electrode was placed on the palm of the hands, while reference electrode was placed on the dorsum of the hand, and the ground electrode on the wrist. A single electrical stimulus ranged from 12 to $20 \mathrm{~mA}$ with a pulse width of $0.1 \mathrm{~ms}$ was given unexpectedly to the median nerve opposite the recorded side. To avoid any habituation, the inter-stimulus interval was between 20 and 30 s. Five responses were recorded to document the reproducibility and the response with minimum latency (the time required to reach the initiation of the first deflection of the wave) was used for further analysis [17].

Interictal EEG was recorded before the autonomic function testing, using a video-EEG monitoring system (Nicolet ${ }^{\mathrm{TM}}$ Viking Quest, Italy); the electrodes were arranged according to the International 10-20 system. The duration of video-EEG is at least $3 \mathrm{~h}$. All patients were partially sleep deprived; and the usual dose of their AEDs was regularly taken.

Statistical analysis was performed using IBM-SPSS (statistical package for Social Sciences) version 25 (IBM Corporation, USA). Quantitative variables were presented as mean \pm standard deviation (SD) and analyzed with independent Student's $t$-test. Categorical variables were expressed as counts and percentages and analyzed with Chi-square test. Correlations between different quantitative variables were performed with two-tailed Pearson's correlation analysis. For all the tests, a significant level of statistics was considered when $p<0.05$.

\section{Results}

The demographic data of study population are presented in Table 1. In terms of age and gender between PwE and controls, there are no statistical difference. Twenty-five $(80 \%)$ of the patients with TLE have orthostatic hypotension, 28 (90\%) have cutaneous manifestation, and 10 (32.2\%) have gastrointestinal symptoms. Meanwhile, 6 (35\%) of the patients with IGE have orthostatic hypotension, 8 (47\%) have excessive sweating, and the rest 12 patients showed no symptoms. When the frequency of

Table 1 Demographic baseline data of the study population

\begin{tabular}{llll}
\hline Characteristic & PwE $(\boldsymbol{n}=\mathbf{4 8})$ & Controls $(\boldsymbol{n}=\mathbf{5 1})$ & P-value \\
\hline Age, years & & & \\
$\quad$ Mean \pm SD & $35.6 \pm 13.11$ & $36.55 \pm 12.7$ & 0.716 \\
Gender & & & \\
$\quad$ Male, $n \%$ & $22(45.8 \%)$ & $22(43.1 \%)$ & 0.787 \\
Female, $n \%$ & $26(54.2 \%)$ & $29(56.9 \%)$ & \\
Type of epilepsy & & & \\
TLE, $n \%$ & $31(64.6)$ & & \\
GE, $n \%$ & $17(35.4)$ & & \\
GE subtypes & & & \\
GME, n\% & $8(47.1)$ & & \\
IGE, $n \%$ & $7(41.2)$ & & \\
JAE, $n \%$ & $2(11.8)$ & & \\
Disease duration (years) & & \\
Mean \pm SD & $7.67 \pm 3.7$ & & \\
Autonomic symptoms & & \\
Yes, $n \%$ & $36(75)$ & & \\
No, $n \%$ & $12(25)$ & $35(68.6)$ & \\
Autonomic symptoms & & \\
TLE, $n \%$ & $28(90.3)$ & & \\
GE, $n \%$ & $8(47.1)$ & & \\
Autonomic function abnormalities & & \\
TLE, $n \%$ & $22(70.97)$ & & \\
GE, $n \%$ & $8(47.1)$ & & \\
\hline
\end{tabular}

PWE people with epilepsy, TLE temporal lobe epilepsy, GE generalized epilepsy, GME generalized myoclonic epilepsy, IGE idiopathic generalized epilepsy, JAE juvenile absence epilepsy 
ANS symptoms of the total PwE was compared with that of the controls, it was significantly higher in the patients in the interictal period $(p<0.001)$. A comparison of the frequency of ANS symptoms in patients with TLE and those with IGE, the former group showed significantly higher frequency $(p=0.001)$.

The systolic and diastolic PB response to active standing differences was significantly higher in $\mathrm{PwE}$ compared to healthy subjects $(p<0.001)$. No significant difference was demonstrated in $\mathrm{R}-\mathrm{R}$ interval during rest between the $\mathrm{PwE}$ and controls $(p=0.582)$. Meanwhile, $\mathrm{R}-\mathrm{R}$ interval during deep breathing, Valsalva maneuver, and during active standing where significantly lower in $\mathrm{PwE}$ as compared to control group $(p=0.012 ; p<0.01$; and $p=0.001$, respectively) (Table 2).

The diastolic BP response to isometric handgrip and the systolic BP response to mental stress test were significantly lower $(p<0.001)$ in PwE in comparison to the controls. Also, the SSR latency was significantly prolonged $(p<0.001)$ in PwE as compared to the controls (Table 2).

Table 3 illustrates the differences between patients with TLE and IGE. Apart from significant difference in age $(p<0.001)$, none of the other parameters were significantly different between the two epileptic groups.

Although patients with TLE showed more reduction in the $R-R$ interval ratios and more prolongation in SRR latencies, yet, no significant difference was demonstrated between the right and left TLE neither with the demographic data nor with the different autonomic function tests (Table 4).

Figure $1 \mathrm{~A}$ demonstrates negative correlation between the SSR latency and R-R interval during deep breathing $(r=-0.326, p=0.024)$. Moreover, the SSR latency was negatively correlated with $\mathrm{R}-\mathrm{R}$ interval during rest

Table 2 Autonomic function tests in PwE versus controls

\begin{tabular}{lccc}
\hline Variables & PwE $(\boldsymbol{n}=\mathbf{4 8})$ & Controls $(\boldsymbol{n}=\mathbf{5 1})$ & $\boldsymbol{p}$-value \\
\hline R-R interval & & & \\
$\quad$ Rest & $1.54 \pm 0.43$ & $1.5 \pm 0.36$ & $>0.05$ \\
$\quad$ Deep breathing & $1.26 \pm 0.43$ & $1.47 \pm 0.36$ & $<0.01$ \\
$\quad$ Valsalva maneuver & $1.36 \pm 0.32$ & $1.81 \pm 0.25$ & $<0.001$ \\
$\quad$ Standing & $1.07 \pm 0.3$ & $1.23 \pm 0.16$ & $<0.005$ \\
BP to standing (mmHg) & & & \\
$\quad$ During systole & $15.77 \pm 4.12$ & $11.82 \pm 4.07$ & $<0.001$ \\
$\quad$ During diastole & $8.54 \pm 2.94$ & $5.14 \pm 3.12$ & $<0.001$ \\
BP to hand grip (mmHg) & $9.94 \pm 3.63$ & $13.61 \pm 3.3$ & $<0.001$ \\
BP to mental stress test & $14.9 \pm 3.78$ & $18.08 \pm 2.44$ & $<0.001$ \\
(mmHg) & & & \\
SSR latency (s) & $1.78 \pm 0.43$ & $1.39 \pm 0.14$ & $<0.001$ \\
\hline
\end{tabular}

$P W E$ people with epilepsy, $B P$ blood pressure, SSR sympathetic skin response; the data are presented in mean $\pm S D$
Table 3 Autonomic function tests in different types of epilepsy

\begin{tabular}{lccc}
\hline Variables & \multicolumn{2}{c}{ Epilepsy type } & p-value \\
\cline { 2 - 3 } & TLE $(\boldsymbol{n}=\mathbf{3 1})$ & $\mathbf{G E}(\boldsymbol{n}=\mathbf{1 7})$ & \\
\hline R-R interval & & & \\
$\quad$ Rest & $1.50 \pm 0.47$ & $1.62 \pm 0.36$ & 0.353 \\
$\quad$ Deep breathing & $1.33 \pm 0.61$ & $1.42 \pm 0.57$ & 0.643 \\
$\quad$ Valsalva maneuver & $1.33 \pm 0.29$ & $1.42 \pm 0.36$ & 0.399 \\
$\quad$ Standing & $1.03 \pm 0.28$ & $1.14 \pm 0.33$ & 0.224 \\
BP to standing (mmHg) & & & \\
$\quad$ During systole & $16.35 \pm 4.1$ & $13.65 \pm 3.87$ & 0.187 \\
$\quad$ During diastole & $8.94 \pm 3.08$ & $7.82 \pm 2.72$ & 0.219 \\
BP to hand grip (mmHg) & $9.55 \pm 3.7$ & $10.65 \pm 3.5$ & 0.321 \\
BP to mental stress test (mmHg) & $14.26 \pm 3.8$ & $16.06 \pm 3.56$ & 0.116 \\
SSR latency (s) & $1.83 \pm 0.42$ & $1.69 \pm 0.44$ & 0.283
\end{tabular}

Data are presented with mean $\pm S D$

$T L E$ temporal lobe epilepsy, GE generalized epilepsy, BP blood pressure, SSR sympathetic skin response

$(r=-0.343, p=0.017)$ as illustrated in Fig. 1b. Furthermore, the SSR latency was also negatively correlated $(r=-0.332, p=0.024)$ with $\mathrm{R}-\mathrm{R}$ interval during Valsalva maneuver (Fig. 1C).

\section{Discussion}

Generally, 75\% of our PwE demonstrate interictal autonomic symptoms. These autonomic manifestations were frequently seen by other researchers $[6,18-20]$. They are often seen in TLE as well as during or after IGE seizures [19-21]. The mechanisms responsible for these interictal autonomic changes are not fully understood; however, it

Table 4 Autonomic function tests in TLE

\begin{tabular}{lccc}
\hline Variables & \multicolumn{2}{l}{ TLE } & p-value \\
\cline { 2 - 3 } & Right $(\boldsymbol{n}=\mathbf{1 2})$ & Left $(\boldsymbol{n}=\mathbf{1 9})$ & \\
\hline Age (years) & $40.25 \pm 12.68$ & $40.63 \pm 13.0$ & 0.937 \\
$\begin{array}{l}\text { R-R interval } \\
\quad \text { Rest }\end{array}$ & $1.55 \pm 0.26$ & $1.47 \pm 0.57$ & 0.667 \\
$\quad$ Deep breathing & $1.25 \pm 0.38$ & $1.23 \pm 0.48$ & 0.915 \\
$\quad$ Valsalva maneuver & $1.32 \pm 0.28$ & $1.34 \pm 0.29$ & 0.798 \\
$\quad$ Standing & $1.0 \pm 0.13$ & $0.99 \pm 0.29$ & 0.953 \\
BP to standing (mmHg) & & & \\
$\quad$ During systole & $19.0 \pm 6.3$ & $19.1 \pm 5.44$ & 0.961 \\
$\quad$ During diastole & $11.0 \pm 4.88$ & $10.32 \pm 4.81$ & 0.706 \\
BP to hand grip (mmHg) & $8.75 \pm 2.96$ & $10.05 \pm 4.09$ & 0.348 \\
BP to mental stress test & $13.92 \pm 4.23$ & $14.47 \pm 3.6$ & 0.695 \\
(mmHg) & & & \\
SSR latency (s) & $1.92 \pm 0.53$ & $1.78 \pm 0.34$ & 0.370 \\
\hline
\end{tabular}

The data are presented in mean \pm SD

$T L E$ temporal lobe epilepsy, $B P$ blood pressure, $S S R$ sympathetic skin response 

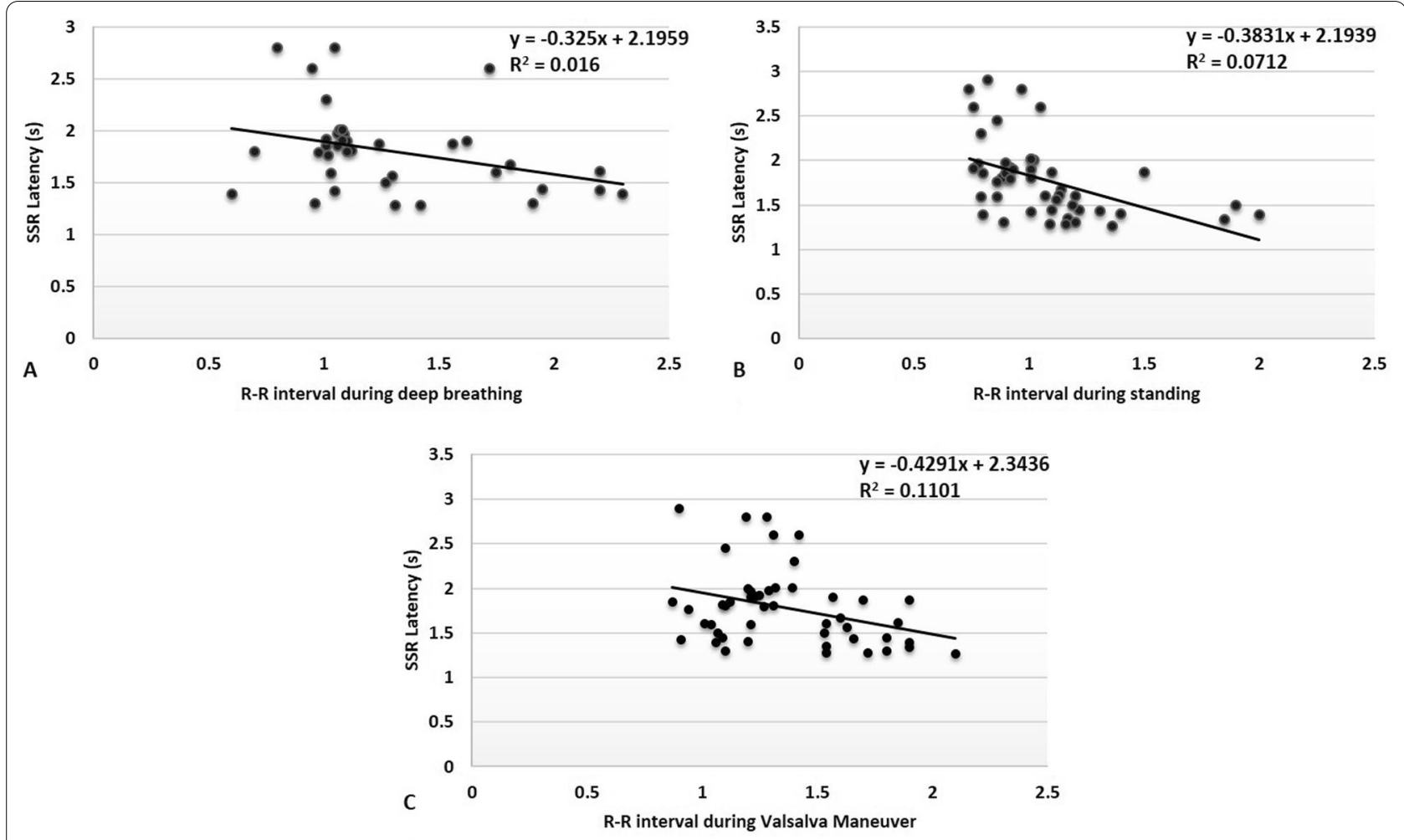

Fig. 1 Regression line of SSR latency and R-R interval during deep breathing (A), and R-R interval during rest (B), and $R-R$ interval during Valsalva maneuver in patients with epilepsy (C)

has been postulated that gradual changes may occur in the CANs from repeated epileptogenic insults arising or spreading to these areas [22, 23].

In patients affected by TLE, the frequency of ANS symptoms was significantly higher than those with IGE. This is because the temporal lobe plays a central role in the activity of the CAN. Moreover, experimental data indicate that ictal and interictal epileptogenic activity can spread from the temporal lobe and interfere with autonomic functions. Furthermore, chronic TLE can induce structural lesions characterized by the presence of significant neuronal loss and sclerosis in two regions that are involved in the CAN, like the amygdala and the hippocampus [24, 25].

In our study, the percentage of seizures during which there is interictal autonomic change appears to be high during temporal lobe seizures than extra-temporal seizures. This finding was also reported by many other studies [26-28].

The interictal $\mathrm{R}-\mathrm{R}$ interval and $\mathrm{BP}$ changes of PwE of this study indicate ANS (parasympathetic and sympathetic) dysregulation. Such changes denote an interrelationship between the brain and the heart in PwE [4, 14, 29, 30]. These epilepsy-related cardiac manifestations can occur ictally, but also in the interictal period [11, 31]. This has been shown in various types of epilepsy, including TLE and IGE $[32,33]$.

The concept of the epileptic heart was proposed recently "repeated rises in catecholamines and hypoxemia during chronic epilepsy causes injury to the heart and coronary vasculature resulting in both electrical and mechanical dysfunction [34, 35]".

SSR latency but not the SSR amplitude was evaluated in our PwE because the SSR latency values are less affected by the habituation phenomenon, therefore they are more objectively reflect ANS dysfunction [36]. The latencies were longer in the upper extremity of PwE when compared to the control group. SSR latency is suggested to reflect the conduction of the efferent sudomotor pathway and the postganglionic non-myelinated $C$ fibers [37]. Similar to our results, Drake et al. [38] and Berilgen et al. [39] also reported prolonged SSR latencies in epileptic patients. Conversely, Atalar et al. [14] found no difference in the SSR latency between PwE and controls.

It is well documented that the SSR has an excitatory suprasegmental input from cerebral cortex and inhibitory inputs from the striatum reflecting the activity of posterior hypothalamus and brainstem reticular formation 
[38]. Such centers are affected by seizures and interictal paroxysms and may play a role in epilepsy-related autonomic symptoms. So, it is rational for the SSR to reflect the effects of central influences on autonomic function.

Many studies documented prolonged SSR latencies in diseases where the ANS is extensively involved such as Parkinson's disease, complex regional pain syndrome, and amyotrophic lateral sclerosis, and ischemic strokes [30, 40-42].

None of the autonomic function tests was different between the two disease subtypes. Despite the exclusion criteria of the present study, factors such as the small sample size of the two subgroups, cognitive status of the patients, patient compliance with examinations, nature of epilepsy disease, and probable subclinical seizures may have affected our outcomes.

In this study, TLE patients were differentiated by the right or left location of the epileptic foci to assess the relationship between interictal epileptiform discharges and modifications of the autonomic cardiac and sudomotor control. Our data show no side-specific hemispheric lateralization between left TLE and right TLE in all studied cardiovascular autonomic and sudomotor tests indicating loss of lateralized control of autonomic functions in PwE. Conversely, several studies suggest hemispheric lateralization of autonomic cardiovascular control [43-45].

The divergence of our study from other studies for the absent lateralization may be due to the fact that irrespective of the localization and the onset of epileptic discharges, they may spread and activate autonomic centers. Moreover, chronicity of disease, refractoriness to treatment, and continuous seizures that worsen the ANS functions in both right and left TLE patients. Thirdly, influence of the testing method used in this study to investigate autonomic function as majority of other studies test the HRV with 24-h electrocardiogram monitoring.

The negative correlation between sudomotor test (SSR latency) and the cardiovascular (R-R interval) in PwE of this study may indicate that both sympathetic and parasympathetic divisions of ANS are affected in the same way.

Despite that our PwE were on AEDs (bi- or polytherapy) which could denote to major limitation. A possible detrimental effect of AEDs on electrical properties of cardiac cells and thereby the HRV therefore cannot be completely excluded. Nevertheless, the role of AEDs in the causation of cardiac autonomic neuropathy is controversial. Some authors have reported that carbamazepine use may aggravate autonomic dysfunction in epilepsy patients, whereas others have found no relationship between AED treatment and interictal autonomic dysfunction [8, 11, 39, 46, 47]. The absent differences in all autonomic tests between patients with TLE (majority of them on carbamazepine) and patients with IGE (treated with sodium valproate) would minimize the effect of AEDs on the results of this study.

\section{Conclusions}

Abnormal autonomic (sympathetic and parasympathetic) regulatory functions suggest that epilepsy may alter the autonomic function and this is not only in TLE but rather in IGE too. These autonomic changes are irrespective of the localization of epilepsy between the two hemispheres. The ANS changes in epileptic patients, particularly those with autonomic symptoms, confirm that electrophysiologic measures of autonomic function may be of value in preventing sudden unexpected death due to epilepsy.

\section{Abbreviations \\ AEDs: Antiepileptic drugs; ANS: Autonomic nervous system; BP: Blood pres- sure; CAN: Central autonomic network; EEG: Electroencephalography; HRV: Heart rate variability; IGE: Idiopathic generalized epilepsy; MRI: Magnetic resonance imaging; PwE: People with epilepsy; SD: Standard deviation; SSR: Sympathetic skin response; TLE: Temporal lobe epilepsy.}

\section{Acknowledgements}

We thank assistant professor Dr. Qasim Al-Mayah from the Research Medical Unit/College of Medicine/Al-Nahrain University for helping in statistical analysis.

\section{Authors' contributions}

All the authors have directly participated in the preparation of this manuscript and have approved the final version submitted. AA clinically examined and referring people with epilepsy. KS and FH did the electrodiagnostic tests. $\mathrm{FH}$ and $\mathrm{KS}$ drafted the manuscript. AA, KS, and FH conceived the study and participated in its design and interpretation. All authors read and approved the final manuscript.

\section{Funding}

This research did not receive any specific grant from funding agencies in the public, commercial, or not-for-profit sectors.

\section{Availability of data and materials}

All data generated or analyzed during this study are included in this published article. The datasets used and/or analyzed during the current study are available from the corresponding author on reasonable request.

\section{Declarations}

\section{Ethics approval and consent to participate}

The study was approved by the Iraqi Board for Medical Specialization (Order No. 931: date: 1/3/2020. Written consent for participation from all subjects was ensured.

\section{Consent for publication \\ Not applicable.}

\section{Competing interests}

The authors declare no competing interests.

\section{Author details}

${ }^{1}$ Neurophysiology Unit, Al-Imamain Al-Kadhimiyian, Medical City, Baghdad, Iraq. ${ }^{2}$ Department of Neurology, Baghdad Teaching Hospital, Medical City, 
Baghdad, Iraq. ${ }^{3}$ Department of Physiology, College of Medicine, Al-Nahrain University, P.O. Box 70044, Baghdad, Iraq.

Received: 10 August 2021 Accepted: 24 November 2021

Published online: 07 December 2021

\section{References}

1. Thijs RD, Surges R, O'Brien TJ, Sander JW. Epilepsy in adults. Lancet. 2019:393:689-701

2. Falco-Walter JJ, Scheffer IE, Fisher RS. The new definition and classification of seizures and epilepsy. Epilepsy Res. 2018;139:73-9.

3. Steinlein OK. Genetic mechanisms that underlie epilepsy. Nat Rev Neurosci. 2004;5:400-4083.

4. Shmuely S, van der Lende M, Lamberts RJ, Sander JW, Thijs RD. The heart of epilepsy: current views and future concepts. Seizure. 2017:44:176-83.

5. Goit RK, Jha SK, Pant BN. Alteration of cardiac autonomic function in patients with newly diagnosed epilepsy. Physiol Rep. 2016;4: e12826.

6. Baumgartner C, Koren J, Britto-Arias M, Schmidt S, Pirker S. Epidemiology and pathophysiology of autonomic seizures: a systematic review. Clin Auton Res. 2019:29:137-50.

7. Sivathamboo S, Perucca P. Interictal autonomic dysfunction. Curr Opin Neurol. 2021:34(2):197-205.

8. Ansakorpi H, Korpelainen JT, Suominen K, Tolonen U, Myllylä W, Isojärvi Jl. Interictal cardiovascular autonomic responses in patients with temporal lobe epilepsy. Epilepsia. 2000;41(1):42-7.

9. Persson $\mathrm{H}$, Kumlien E, Ericson M, Tomson T. Circadian variation in heart-rate variability in localization-related epilepsy. Epilepsia. 2007:48(5):917-22.

10. Ansakorpi H, Korpelainen JT, Huikuri HV, Tolonen U, Myllylä W, Isojärvi J. Heart rate dynamics in refractory and well controlled temporal lobe epilepsy. J Neurol Neurosurg Psychiatry. 2002;72(1):26-30.

11. Sathyaprabha TN, Satishchandra P, Netravathi K, Sinha S, Thennarasu K, Raju TR. Cardiac autonomic dysfunctions in chronic refractory epilepsy. Epilepsy Res. 2006;72:49-56.

12. Müngen B, Berilgen MS, Arıkanoğlu A. Autonomic nervous system functions in interictal and postictal periods of nonepileptic psychogenic seizures and its comparison with epileptic seizures. Seizure. 2010;19(5):269-73.

13. Ponnusamy A, Marques JL, Reuber M. Comparison of heart rate variability parameters during complex partial seizures and psychogenic nonepileptic seizures. Epilepsia. 2012;53:1314-21.

14. Atalar Al, Savrun FK, Yeni SN. Autonomic dysfunction during the interictal period: an electrophysiologic study. Neurol Sci Neurophysiol. 2019;36(1):9-15.

15. Zygmunt A, Stanczyk J. Methods of evaluation of autonomic nervous system function. Arch Med Sci. 2010;6:11.

16. Ewing DJ, Martyn CN, Young RJ, et al. The value of cardiovascular autonomic function tests: 10 years-experience in diabetes. Diabetes Care. 1985:8:491-8.

17. Dumitru D, Zwarts MJ. Special nerve conduction techniques. In: Electrodiagnostic medicine. 2nd ed. Philadelphia: Hanly and Belfus; 2002. p. 252.

18. Freeman R. Cardiovascular manifestations of autonomic epilepsy. Clin Auton Res. 2006;16:12-7.

19. Janszky J, Fogarasi A, Toth V, Magalova V, Gyimesi C, Kovacs N, Schulz R, Ebner A. Peri-ictal vegetative symptoms in temporal lobe epilepsy. Epilepsy Behav. 2007:11:125-9.

20. Baysal-Kirac L, Baykan B. Current view of autonomic findings during epileptic seizures. Turk J Neurol. 2015:21:147-53.

21. Moseley BD, Bateman L, Millichap JJ, Wirrell E, Panayiotopoulos CP. Autonomic epileptic seizures, autonomic effects of seizures, and SUDEP. Epilepsy Behav. 2013;26:375-85.

22. Sevcencu C, Struijk JJ. Autonomic alterations and cardiac changes in epilepsy. Epilepsia. 2010;51(5):725-37.

23. Mukherjee S, Tripathi M, Chandra PS, Yadav R, Choudhary N, Sagar R, et al. Cardiovascular autonomic functions in well-controlled and intractable partial epilepsies. Epilepsy Res. 2009;85:261-9.

24. Babb TL, Brown WJ, Pretorius J, Davenport C, Lieb JP, Crandall PH. Temporal lobe volumetric cell densities in temporal lobe epilepsy. Epilepsia. 1984;25:729-40

25. Kandratavicius L, Balista P, Lopes-Aguiar C, Ruggiero R, Umeoka E, GarciaCairasco N, et al. Animal models of epilepsy: use and limitations. Neuropsychiatr Dis Treat. 2014;10:1693-705.
26. Leutmezer F, Schernthaner C, Lurger S, Pötzelberger K, Baumgartner C. Electrocardiographic changes at the onset of epileptic seizures. Epilepsia. 2003:44:348-54

27. Weil S, Arnold S, Eisensehr I, Noachtar S. Heart rate increase in otherwise subclinical seizures is different in temporal vs. extratemporal seizure onset: support for temporal lobe autonomic influence. Epileptic Disord. 2005;7:199-204

28. Eggleston KS, Olin BD, Fisher RS. Ictal tachycardia: the head-heart connection. Seizure. 2014;23:496-505.

29. Myers KA, Sivathamboo S, Perucca P. Heart rate variability measurement in epilepsy: how can we move from research to clinical practice. Epilepsia. 2018:59:2169-78.

30. Wang J-Y, Wang M-Y, Liu R-P, Li Y, Zhang W-Y, Ovlyakulov B, et al. Association analyses of autonomic dysfunction and sympathetic skin response in motor subtypes of Parkinson's disease. Front Neurol. 2020;11:577128.

31. Laure M, Sylvain R. Ictal and interictal cardiac manifestations in epilepsy. A review of their relation with an altered central control of autonomic functions and with the risk of SUDEP. Front Neurol. 2021;12:329.

32. Massetani R, Strata G, Galli R, Gori S, Gneri C, Limbruno U, et al. Alteration of cardiac function in patients with temporal lobe epilepsy: different roles of EEG-ECG monitoring and spectral analysis of RR variability. Epilepsia. 1997:38:363-9.

33. Tomson T, Ericson M, Ihrman C, Lindblad LE. Heart rate variability in patients with epilepsy. Epilepsy Res. 1998;30:77-83.

34. Fialho GL, Wolf P, Walz R, Lin K. Epilepsy and ultra-structural heart changes: the role of catecholaminergic toxicity and myocardial fibrosis. What can we learn from cardiology. Seizure. 2019;71:105-9.

35. Verrier RL, Pang TD, Nearing BD, Schachter SC. The epileptic heart: concept and clinical evidence. Epilepsy Behav. 2020;105: 106946.

36. Devinsky O, Perrine $K$, Theodore WH. Interictal autonomic nervous system function in patients with epilepsy. Epilepsia. 1994;35:199-204.

37. Akyüz G, Akdeniz-Leblebiciler M. Anatomy and assessment of the autonomic nervous system. Turk J Phys Med Rehab. 2012;58(Supp 1):1-5.

38. Drake ME Jr, Andrews JM, Castleberry CM. Electrophysiologic assessment of autonomic function in epilepsy. Seizure. 1998;7(2):91-6.

39. Berilgen MS, Sari T, Bulut S, Mungen B. Effects of epilepsy on autonomic nervous system and respiratory function tests. Epilepsy Behav. 2004;5:513-6.

40. Georgieva-Hristova D. Sympathetic skin response in acute ischemic stroke-hemispheric asymmetry. Scr Sci Med. 2016;48(4):43-7.

41. Keleș BY, Önder B, Kesiktaş FN, Öneș K, Paker N. Acute effects of contrast bath on sympathetic skin response in patients with poststroke complex regional pain syndrome. Somatosen Motor Res. 2020;37(4):320-5.

42. Mahbub Alam SK, Hasan M, Rahman HZ. Motor neuron disease (MND) O-MN001. Association of sympathetic skin response in amyotrophic lateral sclerosis. Clin Neurophysiol. 2021;132(8):e72-3.

43. Koseoglu E, Kucuk S, Arman F, Ersoy AO. Factors that affect interictal cardiovascular autonomic dysfunction in temporal lobe epilepsy: role of hippocampal sclerosis. Epilepsy Behav. 2009;16(4):617-21.

44. Ghchime R, Benjelloun H, Kiai H, Belaidi H, Lahjouji F, Ouazzani R. Cerebral hemispheric lateralization associated with hippocampal sclerosis may affect interictal cardiovascular autonomic functions in temporal lobe epilepsy. Epilepsy Res Treat. 2016;2016: 7417540.

45. Dono F, Evangelista G, Frazzini V, Vollono C, Carrarini C, Russo M, et al. Interictal heart rate variability analysis reveals lateralization of cardiac autonomic control in temporal lobe epilepsy. Front Neurol. 2020;11:842.

46. Katsiki N, Mikhailidis DP, Nair DR. The effects of antiepileptic drugs on vascular risk factors: a narrative review. Seizure. 2014;23:677-84.

47. Izzi F, Placidi F, Liguori C, Posca I, Lauretti B, Diomedi M, et al. Autonomic functions in focal epilepsy: a comparison between lacosamide and carbamazepine monotherapy. J Neurol Sci. 2020;418(15): 117095.

\section{Publisher's Note}

Springer Nature remains neutral with regard to jurisdictional claims in published maps and institutional affiliations. 